\title{
Atypical Pleural Effusions in Patients on Peritoneal Dialysis
}

\author{
Hari Wimaleswaran ${ }^{a}$, Stella Setyapranata ${ }^{\mathrm{a}}$, Paul Champion de Crespigny ${ }^{\mathrm{a}}$, \\ Stephen G. Holt ${ }^{\mathrm{a}, \mathrm{b}, \mathrm{c}}$
}

\begin{abstract}
Rarely, peritoneal dialysis (PD) is complicated by a hydrothorax, often referred to as a pleural effusion, that commonly occurs early after starting PD and is usually right-sided. We illustrate three cases of atypical hydrothorax presenting in PD patients. Case 1 is a 72-yearold lady with left-sided pleuroperitoneal communication confirmed on nuclear imaging. Case 2 is a 50 -year-old lady who presented with right-sided pleuroperitoneal leak which occurred 11 months after commencement of PD. The last case is a 64-year-old man with congestive cardiac failure secondary to ischemic heart disease who had recurrent right-sided pleural effusion despite multiple pleural drainage. He continued on PD with no drainage problems. Pleuroperitoneal leaks need to be excluded even in patients presenting with left-sided effusion and in those who present late after commencement of PD.
\end{abstract}

Keywords: Peritoneal dialysis; Pleuroperitoneal leak; Hydrothorax; Pleural effusion

\section{Introduction}

Peritoneal dialysis (PD) is an excellent initial form of renal replacement therapy, but like all renal replacement modalities coming with a number of infective and non-infective complications, rarely these include hydrothorax in up to $7 \%$ of all PD patients [1]. When this does occur, it is usually right-sided and characteristically occurs early after starting PD. We present three atypical cases of hydrothorax complicating PD.

\section{Case Reports}

\section{Case 1}

A 72-year-old female, with renal failure secondary to reflux

\section{Manuscript accepted for publication August 19, 2015}

\footnotetext{
aThe Royal Melbourne Hospital, Parkville, Victoria, Australia

${ }^{b}$ Department of Medicine, The University of Melbourne, Parkville, Australia

${ }^{\mathrm{c} C}$ Corresponding Author: Steve Holt, Department of Nephrology, Royal Melbourne Hospital, Grattan Street, Parkville, Victoria 3052, Australia.

Email: steve.holt@mh.org.au
}

doi: http://dx.doi.org/10.14740/wjnu227w nephropathy and other comorbidities including cardiac dysfunction, was started on PD. She presented 2 weeks later complaining of increasing dyspnea and peripheral edema, which were managed by increasing her diuretic dose and changes in her dialysis regimen. Her symptoms did not improve and a week later she had a chest X-ray (CXR) (Fig. 1a) showing a left-sided pleural effusion. A technetium-99m DTPA scintigraphy confirmed a left-sided pleuroperitoneal communication (Fig. 1b) with appearance of the tracer in the left pleural space. PD was ceased and she was commenced on hemodialysis and underwent drainage of the pleural fluid. Pleural fluid analysis showed LDH $36 \mathrm{IU}$, glucose $7.1 \mathrm{mmol} / \mathrm{L}$, protein $<8 \mathrm{~g} / \mathrm{L}$ compared to serum values of $287 \mathrm{IU}, 6.1 \mathrm{mmol} / \mathrm{L}$ and $55 \mathrm{~g} / \mathrm{L}$ respectively. The patient had a normal follow-up CXR and no further dyspneic episodes.

\section{Case 2}

A 50-year-old female reached end-stage renal failure from IgA nephropathy and started on PD in August 2013 and did not encounter any problems until February 2014 when she developed constipation. This was treated conservatively with increasing aperients. Shortly after, she encountered more drainage problems and her abdominal X-ray showed a malpositioned PD catheter tip which was located in the right hypochondrial region. Encouraging bowel motility failed to help reposition the catheter back into the pelvis and she underwent laparoscopic repositioning, seemingly without problems. She was re-started on low volume PD, but she had difficulty with PD fluid retention even with icodextrin and started to complain of dyspnea. CXR revealed a right pleural effusion (Fig. 2a, b) and a PD-related hydrothorax was suspected and subsequently confirmed after aspiration. This necessitated removal of the PD catheter and temporary transfer to hemodialysis (HD) before she was recently transplanted.

\section{Case 3}

A 64-year-old man with diabetes and a history of ischemic heart disease with previous extensive cardiac revascularization presented with mildly increased exertional dyspnea 11 months after starting PD. Clinically a large right pleural effusion was noted and confirmed on CXR (Fig. 3) and a leak was suspected. PD scintigraphy could not demonstrate a pleuroperitoneal connection and dialysis was otherwise going reasonably well with no evidence of retained fluid and euvolemia. The effu- 


\section{a}

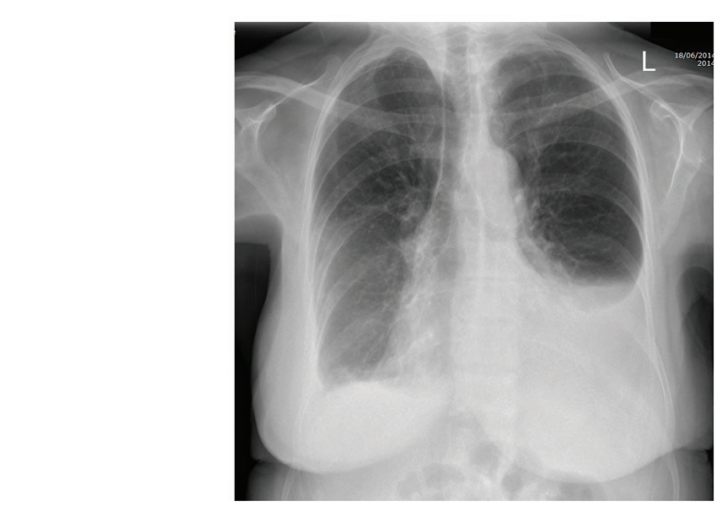

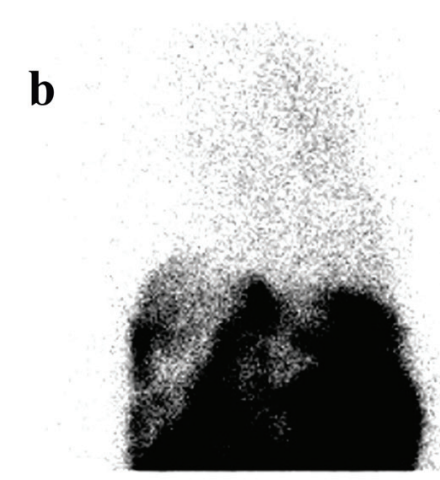

RT

Anterior T0+135MIN
LT

LT

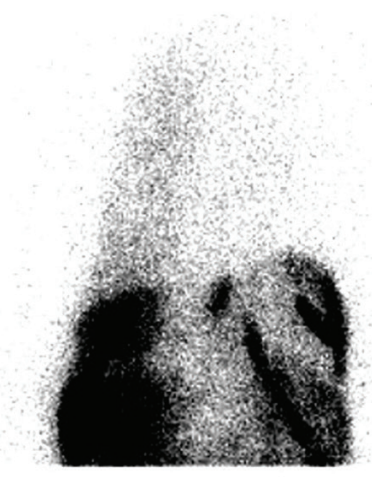

$\mathrm{T}$

Posterior T0+135MIN
RT

Figure 1. (a) Posterior-anterior CXR showing a left-sided pleural effusion. (b) Tc-99m DTPA scintigraphy showing left-sided pleuroperitoneal leak with accumulation of tracer within the left lung space compared with the right side, seen on anterior and posterior views. There is of course a large and expected signal from within the peritoneal space.

sion was drained repeatedly but recurred and on all occasions analysis of the fluid suggested a transudate rather than PD fluid (sodium $133 \mathrm{mmol} / \mathrm{L}$, potassium $5.1 \mathrm{mmol} / \mathrm{L}$, chloride 99 $\mathrm{mmol} / \mathrm{L}$, glucose similar to blood glucose, protein $12-19 \mathrm{~g} / \mathrm{L}$, LDH 50 IU with corresponding plasma values of sodium 138 $\mathrm{mmol} / \mathrm{L}$, potassium $5 \mathrm{mmol} / \mathrm{L}$, chloride $98 \mathrm{mmol} / \mathrm{L}$, glucose $6-17 \mathrm{mmol} / \mathrm{L}$, albumin $25 \mathrm{~g} / \mathrm{L}$, total protein $53 \mathrm{~g} / \mathrm{L}$, and $\mathrm{LDH}$ $275 \mathrm{IU})$. The fluid was clear and cytology showed reactive mesothelial cells with background mixed inflammatory cells. Myocardial perfusion scanning showed severe ischemic cardiomyopathy with left ventricular ejection fraction of about $19 \%$ at rest. A diagnosis of cardiac failure complicated by recurrent right pleural effusion was made and his automated peritoneal dialysis prescription remains unchanged and he remains stable.

\section{Discussion}

Pleuroperitoneal leaks after PD are usually right-sided and often occur early (within a month) after commencement of PD [2].

Determining whether the symptoms of hydrothorax are due to PD leak rather than other conditions may be difficult, as all such patients may complain of dyspnea or orthopnea, although hydrothorax may be asymptomatic in up to $25 \%$ of patients [3]. Other conditions giving rise to isolated pleural effusion include pulmonary infarction, congestive cardiac failure, pericardial disease, neoplasm and pneumonia, all of which may be present in co-morbid patients with renal disease. This is especially important when one considers that there is no pleural effusion side predominance with cardiac failure related effusions [4] and as illustrated in case 3 which can look suspiciously like a pleural leak.

Whilst the diagnosis of hydrothorax due to PD is one that is often presumptive, based upon the time of presentation (shortly after commencement), and the side of the effusion (right), this is not always reliable and we illustrate this by presenting a series of atypical presentations of such cases.

The cause of PD fluid leaks are considered to be mainly due to congenital or acquired diaphragmatic defects, with very occasional disorders of lymphatic drainage or severe overfill with high pleuroperitoneal pressure gradients [5]. The migration of dialysate through acquired defects is said to be unidirectional and can occur as a result of the negative pleural pres- 

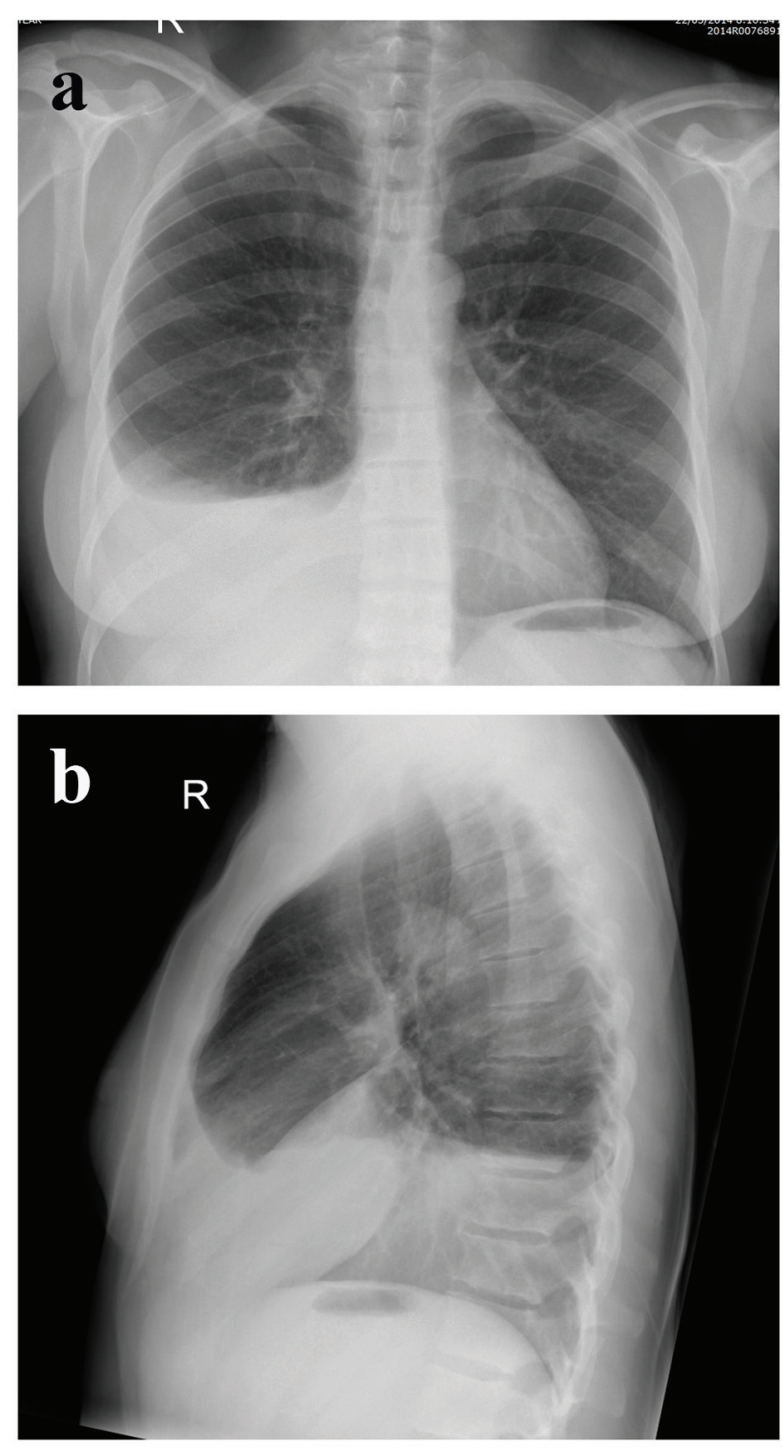

Figure 2. (a) Posterior-anterior. (b) Lateral CXR showing a right anterior basal segment hydrothorax.

sure accompanied with the positive pressure of the peritoneal cavity [6] and congenital defects may exhibit a more bidirectional flow perhaps due to larger size [3]. The fact that similar intra-abdominal problems such as cirrhosis [7], which also usually produce right-sided effusions due to presence of ascitic fluid in the intra-abdominal cavity, suggests that the right side of the chest has an anatomical predisposition for this type of leak and explains why the majority (nearly $90 \%$ ) of these leaks are right-sided [3]. Part of the reason may lie in the fact that the heart and pericardium cover such defects on a large part of the left-sided diaphragmatic surface [3]. However, this may not be the full explanation of the very right-sided predominance of this condition and others have invoked the presence of an embryonic remnant known as the processus vaginalis peritonei, which results in a pneumatoenteric recess connecting the peritoneum with the right side of the chest [8].

Our second case demonstrated that a PD leak occurred

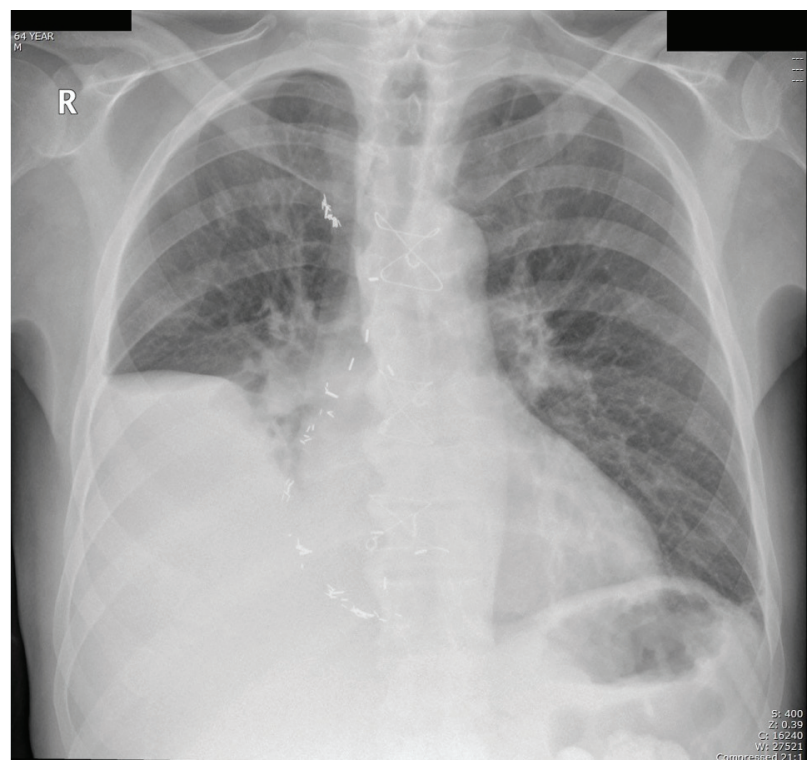

Figure 3. Right pleural effusion related to cardiac failure and not to PD, but note the lack of blunting of the left costophrenic angle.

only after catheter repositioning which involved pneumoperitoneum. This procedure was temporally associated with the development of the pleural leak and we speculate was important in opening a passage between peritoneum and right-sided pneumothorax from pneumoperitoneum, which has been previously reported [9].

A left-sided pleural effusion in PD patients frequently provokes anxiety and a search for another cause of left-sided effusion, especially in the presence of dyspnea [10]. A prompt diagnosis of hydrothorax due to dialysate is required to prevent exacerbation of symptoms and recurrence, but more importantly to prevent unnecessary diagnostic imaging and other procedures as it is so unusual. Other than apparent loss of ultrafiltration [10], investigations that may aid in diagnosis include CXR, paracentesis with analysis of pleural-to-serum glucose and LDH levels, computerized tomography, magnetic resonance, and scintigraphy [11]. Conservative management of the pleuroperitoneal leak has been reported [12] using lower fill volumes or temporary conversion to hemodialysis for a "PD rest". However, in our experience this almost always requires the interruption of $\mathrm{PD}$ and surgical intervention [3]. If $\mathrm{PD}$ is to continue, treatment options include chemical or physical pleurodesis with open thoracotomy or video-assisted thoracoscopic surgery, with success rates of returning to long-term $\mathrm{PD}$ reported in $48-88 \%$ [11].

\section{Conclusion}

Formation of hydrothorax in patients on PD is an uncommon but a well-established complication. Left-sided hydrothorax is particularly uncommon but does occur and should be excluded before other diagnostic possibilities are entertained. However, a large unilateral pleural effusion can be caused by other problems including cardiac failure. Whilst most pleuroperitoneal 
leaks occur shortly after starting PD, later presentation and presentation after pneumoperitoneum may be associated with a new leak. A hydrothorax usually requires surgical intervention if PD is to continue or the switching from this dialysis modality.

\section{Conflict of Interests}

The authors declare that there is no conflict of interests regarding the publication of this paper.

\section{References}

1. Van Dijk CM, Ledesma SG, Teitelbaum I. Patient characteristics associated with defects of the peritoneal cavity boundary. Perit Dial Int. 2005;25(4):367-373.

2. Edwards SR, Unger AM. Acute hydrothorax---a new complication of peritoneal dialysis. JAMA. 1967;199(11):853855.

3. Lew SQ. Hydrothorax: pleural effusion associated with peritoneal dialysis. Perit Dial Int. 2010;30(1):13-18.

4. Woodring JH. Distribution of pleural effusion in congestive heart failure: what is atypical? South Med J. 2005;98(5):518-523.
5. Cho Y, D'Intini V, Ranganathan D. Acute hydrothorax complicating peritoneal dialysis: a case report. J Med Case Rep. 2010;4:355.

6. Juergensen PH, Rizvi H, Caride VJ, Kliger AS, Finkelstein FO. Value of scintigraphy in chronic peritoneal dialysis patients. Kidney Int. 1999;55(3):1111-1119.

7. Lieberman FL, Hidemura R, Peters RL, Reynolds TB. Pathogenesis and treatment of hydrothorax complicating cirrhosis with ascites. Ann Intern Med. 1966;64(2):341351 .

8. Gagnon RF, Daniels E. The persisting pneumatoenteric recess and the infracardiac bursa: possible role in the pathogenesis of right hydrothorax complicating peritoneal dialysis. Adv Perit Dial. 2004;20:132-136.

9. Ross J, Farber JE. Right-sided spontaneous pneumothorax complicating therapeutic pneumoperitoneum. Am Rev Tuberc. 1951;63(1):67-75.

10. Ramaema DP, Mpikashe P. Pleuroperitoneal leak: an unusual cause of acute shortness of breath in a peritoneal dialysis patient. Case Rep Radiol. 2014;2014:614846.

11. Chow KM, Szeto CC, Li PK. Management options for hydrothorax complicating peritoneal dialysis. Semin Dial. 2003;16(5):389-394.

12. Szeto CC, Chow KM. Pathogenesis and management of hydrothorax complicating peritoneal dialysis. Curr Opin Pulm Med. 2004;10(4):315-319. 